\title{
BMJ Open Association between infertility treatment and intrauterine growth: a multilevel analysis in a retrospective cohort study
}

Satoshi Shinohara (D) , ${ }^{1}$ Shuji Hirata, ${ }^{1}$ Kohta Suzuki (D) ${ }^{2}$

To cite: Shinohara S, Hirata S, Suzuki K. Association between infertility treatment and intrauterine growth: a multilevel analysis in a retrospective cohort study. BMJ Open 2020;10:e033675. doi:10.1136/ bmjopen-2019-033675

- Prepublication history for this paper is available online. To view these files, please visit the journal online (http://dx.doi. org/10.1136/bmjopen-2019033675).

Received 16 August 2019 Revised 22 February 2020 Accepted 03 April 2020
Check for updates

(c) Author(s) (or their employer(s)) 2020. Re-use permitted under CC BY-NC. No commercial re-use. See rights and permissions. Published by BMJ.

${ }^{1}$ Department of Obstetrics and Gynecology, Yamanashi Daigaku Igakubu, Chuo, Yamanashi, Japan

${ }^{2}$ Department of Health and Psychosocial Medicine, Aichi Medical University School of Medicine, Nagakute, Aichi, Japan

Correspondence to Professor Kohta Suzuki; kohtas@aichi-med-u.ac.jp

\section{ABSTRACT}

Objectives This study aimed to identify intrauterine growth differences according to infertility treatment compared with spontaneous conception and to describe intrauterine growth trajectories.

Design Retrospective cohort study.

Setting A single primary and tertiary medical centre in Japan.

Participants This study included singleton pregnant women with prenatal check-ups and delivery at the University of Yamanashi Hospital between 1 July 2012 and 30 September 2017. Patients were divided into four groups: spontaneous conception, infertility treatment without assisted reproductive technology (ART), freshembryo transfer and frozen embryo transfer (FET). Interventions Differences in intrauterine growth according to the infertility treatment, including ART, and birth weight were evaluated. Multilevel analysis was employed to evaluate intrauterine growth trajectories stratified by the sex of the offspring.

Primary outcome measure Estimated fetal weight (EFW) assessed by ultrasound examination.

Results We assessed data from 37239 prenatal examination results from 2377 pregnant women (spontaneous conception, $\mathrm{n}=1764$; infertility treatment without ART, $n=171$; fresh-embryo transfer, $n=112$; and FET, $\mathrm{n}=330$ ) in the final analysis. Multilevel analysis was adjusted for gestation duration, gestation period, parity, hypertensive disorders of pregnancy, type of infertility treatment, maternal age, smoking status, placenta previa, thyroid disease, gestational diabetes mellitus and the interaction between each potential confounding factor and gestation duration. In male fetuses, the interaction between FET and gestational duration (estimate: 0.36 ; $95 \% \mathrm{Cl}: 0.06$ to 0.67 ) significantly affected the EFW. Similarly, in female fetuses, FET (estimate: -69.85 ; 95\% Cl: -112.09 to -27.61$)$ and the interaction between FET and gestation duration (estimate: $0.57 ; 95 \% \mathrm{Cl}$ : 0.28 to 0.87 ) significantly affected the EFW.

Conclusions This study shows that FET affects intrauterine growth trajectory from the second trimester to term, particularly in female fetuses. Our findings require further prospective research to examine the effect of infertility treatment on fetal growth.

\section{BACKGROUND}

Infertility is defined as a failure to conceive after 12 months of regular and unprotected
Strengths and limitations of this study

This is the first study to evaluate the association between infertility treatments and intrauterine growth using multilevel analyses

- This was a single-centre study and requires further validation in the general population from a largescale and multicentre cohort study.

- Considering the characteristics of longitudinal estimated fetal weight at multiple prenatal check-ups throughout gestation, we performed multilevel analysis with a relatively large sample size.

- Data on gestational weight gain, intake of alcohol and caffeine, antiphospholipid syndrome and socioeconomic status, which may affect intrauterine growth, were not considered in this study.

sexual intercourse ${ }^{1}$ and is estimated to affect one in six couples globally. Treatments available for infertility include ovulation induction with timed intercourse, artificial insemination, in vitro fertilisation (IVF) and intracytoplasmic sperm injection. ${ }^{1}$

Embryo transfer during IVF or intracytoplasmic sperm injection (called assisted reproductive technology (ART) procedures) can be performed using either fresh-thawed or frozen-thawed embryos. Recently, the number of infants born from frozen embryo transfer (FET) has increased compared with those born from fresh-embryo transfer techniques. $^{2-4}$

Several recent studies report an association between birth weight and infertility treatment. For example, ovulation induction and fresh-embryo transfer are associated with an increased risk of small-for-gestational-age and lower birth weight compared with spontaneous conception. ${ }^{5-8}$ As the use of FET is going to increase in the future, it is important to examine the association between FET and fetal growth. Several studies suggest that FET is associated with a higher birth weight 
than spontaneous, fresh-embryo transfer and non-ART conceptions, ${ }^{6}$ as well as with large-for-gestational-age neonates. ${ }^{9-11}$

Knowledge about when and how infertility treatment affects fetal growth might help predict its effect. This might also help us better understand the short-term and long-term prognoses of infertility treatments. ${ }^{12}$ However, to the best of our knowledge, there is no study that has evaluated the effect of various infertility treatments, including FET, on intrauterine growth in detail. Although previous studies report the role of perinatal factors on birth weight, we also explored their role in the trajectory of fetal growth because the effect might be different depending on the stage of pregnancy.

The present study has the following aims; (1) to identify differences in intrauterine growth according to infertility treatment compared with spontaneous conception and (2) to describe intrauterine growth trajectories following different methods of conception by using multi-level models that might be appropriate for statistical analysis using repeated longitudinal measures data in the same individual.

\section{METHODS}

\section{Study design and population}

This retrospective cohort study was conducted at the University of Yamanashi Hospital. The study population comprised of singleton pregnant women who underwent prenatal check-ups and delivered at the University of Yamanashi Hospital between 1 July 2012 and 30 September 2017. Prenatal check-ups and ultrasound examinations were conducted according to the following schedule; once a month from 12 to 23 weeks, every 2 weeks from 24 to 35 weeks, once a week from 36 to 39 weeks and twice a week after 40 weeks. ${ }^{13}$ The pregnant women who were hospitalised for various reasons, such as hypertensive disorders of pregnancy (HDP), placenta previa, gestational diabetes mellitus (GDM) and threatened preterm labour, had prenatal check-ups and ultrasound examinations once a week or more, regardless of the hospitalisation period.

This study was initiated in July 2018. Based on the national ethical guidelines, we have published information on the conduct and purpose of this research on the university's website. Moreover, we have ensured rejection opportunities wherever possible through the university's website. However, none of the patients denied their participation.

\section{Data collection}

Data on baseline demographics, infertility treatment and medical and family history were collected from medical records. The data selected included maternal age at delivery, parity, gestational period, HDP, GDM, pregestational weight status, placenta previa, thyroid disease and smoking status. Most of these factors have been previously described as potential confounding factors for intrauterine growth. ${ }^{13-17}$ Type of infertility treatment was subdivided into spontaneous conception, infertility treatment without ART, fresh-embryo transfer and FET. Infertility treatment without ART included stimulating ovulation with fertility drugs and intrauterine insemination.

HDP was defined as blood pressure $\geq 140 / 90 \mathrm{~mm} \mathrm{Hg}$ on at least two occasions during pregnancy. ${ }^{13} 18$ Prepregnancy body mass index (BMI) was calculated according to the WHO standards (body weight $(\mathrm{kg}) /$ height $\left.(\mathrm{m})^{2}\right)$. We classified the participants as being underweight (BMI $<18.5 \mathrm{~kg} / \mathrm{m}^{2}$ ), normal (BMI $18.5-25.0 \mathrm{~kg} / \mathrm{m}^{2}$ ), or overweight (BMI $\left.\geq 25.0 \mathrm{~kg} / \mathrm{m}^{2}\right)$ in accordance with the WHO classification of BMI. ${ }^{19}$ We categorised maternal age as $<25,25-34$, or $\geq 35$ years. GDM was diagnosed if there was $\geq 1$ abnormal plasma glucose value ( $\geq 92,180$ and $153 \mathrm{mg}$ / $\mathrm{dL}$ for fasting, 1-hour and 2-hour plasma glucose concentrations, respectively) after a 75-gram oral glucose tolerance test. ${ }^{1320}$ Placenta previa was defined as the presence of placental tissue that extended over the internal cervical os. ${ }^{21}$ Thyroid disease was defined as Basedow disease or hypothyroidism (serum thyroid-stimulating hormone (TSH) concentrations $>2.5 \mathrm{mIU} / \mathrm{L}$ in the first trimester and $>3 \mathrm{mIU} / \mathrm{L}$ in the second trimester). ${ }^{22}$ Smoking status was ascertained at the first visit and we divided the participants into two groups: smoking and non-smoking. The gestational age was determined based on the last menstrual period. Moreover, gestational age according to fresh-embryo transfer was calculated from the day of oocyte retrieval, which was converted into menstrual age by adding 14 days. Hormone-supplemented cycle FET was performed 5 days after the administration of progesterone vaginal tablets, and the actual gestational age was calculated by adding 14 days to the date when these tablets were used for the first time. On the contrary, natural cycle FET was performed 3 days after ovulation, and the actual gestational age was calculated by adding 14 days to the ovulation date. Since there were cases introduced from other hospitals due to perinatal complications or as per patient's will, the gestational age was confirmed by ultrasonography in all cases. We calculated the estimated fetal weight (EFW) at each prenatal check-up using the Shinozuka technique, which is commonly used in Japan. ${ }^{23}$ The formula is as follows: EFW $(\mathrm{g})=1.07 \times \mathrm{BPD}^{3}+3.00 \times$ $10^{-1} \mathrm{AC}^{2} \times \mathrm{FL}$, where $\mathrm{BPD}$ stands for biparietal diameter $(\mathrm{cm}), \mathrm{AC}$ stands for abdominal circumference $(\mathrm{cm})$ and FL stands for femur length $(\mathrm{cm}){ }^{23}$

We used EFW to estimate intrauterine growth since BPD, AC and FL do not accurately reflect intrauterine growth. For example, the association between BPD and fetal growth restriction was rarely observed. ${ }^{24}{ }^{25}$ In contrast, AC was the primary parameter associated with impaired intrauterine growth. ${ }^{24}$ This phenomenon may reflect the brain-sparing effect due to endothelial dysfunction. Fetal growth restriction is most commonly caused by placental insufficiency. In response, the fetus adapts its circulation to preserve oxygen and nutrient supply to the brain (brain-sparing). ${ }^{25}$ We divided the gestational 
duration (days) into eight periods because there may be differences in the effects of infertility treatment on intrauterine growth during each period of pregnancy. We built a model equation to consider the difference. The periods were as follows; period 1 (98-104 days), period 2 (105132 days), period 3 (133-160 days), period 4 (161-188 days), period 5 (189-216 days), period 6 (217-244 days), period 7 (245-272 days) and period 8 ( $\geq 273$ days).

\section{Statistical analysis}

Linear mixed-effects regression analysis (random intercepts and slopes model) was conducted to determine the estimates of slopes in each gestational period by infertility treatment since repeated measurement results were used. There are several reasons why this multilevel analysis is helpful for analysis using repeatedly measured data. First, unlike other traditional statistical models, multilevel analysis can handle correlated longitudinal data within an individual level as a nested structure ${ }^{26}$ Second, time can be treated as a continuous variable in multilevel analysis. Thus, unbalanced data and unequal spacing conditions can be flexibly handled under multilevel analysis through the adequate specification of the time predictor. ${ }^{26}$ Finally, although missing data can arise for many reasons in longitudinal research (ie, missed appointments, participant incapacity, dropout, or lost follow-up), missing data can be handled flexibly in the multilevel analysis. ${ }^{26}$ The first level represented repeated measurements clustered within individuals, and the second level was the individual level. As in previous studies, ${ }^{1927-29}$ we constructed the following model to evaluate differences in the intercepts and slopes for each interval between the gestational periods:

$\underset{{ }_{3}+\beta_{6} * \mathrm{X}_{3} * \mathrm{X}_{1 i t}+}{\mathrm{EFW}_{\text {it }}} \quad=\beta_{1}+\beta_{2} * \mathrm{X}_{1 i t}+\beta_{3} * \mathrm{X}_{2}+\quad \beta_{7} * \mathrm{X}_{4}+\beta_{8} * \mathrm{X}_{4} * \mathrm{X}_{1 i t}+\beta_{9} * \mathrm{X}_{5}+\beta_{10}+\beta_{5} * \mathrm{X}-$ $\mathrm{X}_{1 i t}+\beta_{11} * \mathrm{X}_{6}+\beta_{12} * \mathrm{X}_{6} * \quad \mathrm{X}_{1 i t}+\beta_{13} * \mathrm{X}_{7}+\beta_{14} * \mathrm{X}_{7}^{*} \quad \mathrm{X}_{1 i t}+\beta_{15} * \mathrm{X}-$ ${ }_{8}+\beta_{16} * \mathrm{X}_{8} * \mathrm{X}_{1 i t}+\beta_{17} * \mathrm{X}_{9}+\beta_{18} * \mathrm{X}_{9} * \mathrm{X}_{1 i t}+\beta_{19} * \mathrm{X}_{10}+\beta_{20} *$ $\mathrm{X}_{10} * \mathrm{X}_{1 i t}+\beta_{21} * \mathrm{X}_{11}+\beta_{22} * \mathrm{X}_{11} * \mathrm{X}_{1 i t}+e_{i t}+b_{1 i}+b_{2 i} * \mathrm{X}_{1 i t}$

where $i$ represents the individual, $t$ represents time, $\beta 1-22$ represent the regression coefficient of the fixed effect and $e$ is the error term. ( $\mathrm{X}_{1}$, gestational duration; $\mathrm{X}_{2}$, gestational period; $\mathrm{X}_{3}$, multiparity; $\mathrm{X}_{4}, \mathrm{HDP}$; $\mathrm{X}_{5}$, infertility treatment type; $\mathrm{X}_{6}$, maternal age group; $\mathrm{X}_{7}$, smoking status; $\mathrm{X}_{8}$, placenta previa; $\mathrm{X}_{9}$, Basedow disease; $\mathrm{X}_{10}$, hypothyroidism; $\mathrm{X}_{11}$, GDM).

We describe the reference intrauterine growth trajectories for each infertility treatment group (25-34 years old, nulliparity, normal prepregnancy BMI, non-smoking, no pregnancy complications) after controlling for pregnancy complications (HDP, GDM, placenta previa, Basedow disease and hypothyroidism) and factors affecting intrauterine growth (gestational duration, smoking, parity, maternal age and prepregnancy BMI) to better understand the results of the multilevel analysis.

We used the estimated value of the reference group (ie, estimated values are all zero) to describe growth trajectories, with the exception of infertility treatment. The estimated intercept and slope of each gestational period was calculated from the results of the multilevel analysis.
Then, the estimated lines of each period were described using EFW at each gestational week, which was calculated based on these values.

All analyses were performed using IBM SPSS Statistics V.25 and SAS V.9.4.

\section{Patient and public involvement}

Patients were not invited to comment on the study design and were not consulted to develop patient-relevant outcomes or interpret the results.

\section{RESULTS \\ Maternal background}

Among 2583 pregnant women who underwent prenatal check-ups during the study period and delivered at $>22$ weeks gestation (excluding stillbirth), women with twin pregnancies $(\mathrm{n}=38)$ and with missing obstetric information $(n=158)$ were excluded. To increase the homogeneity of our study population, we also excluded patients with a chromosomal abnormality $(n=10)$. Finally, 37239 prenatal examination results from 2377 pregnant women (average 15.6 examinations per woman, 1196 male fetuses $(50.3 \%))$ were included for the final multilevel analysis model. Patients were divided into the following four groups: spontaneously conceived $(n=1764)$, infertility treatment without ART $(n=171)$, fresh-embryo transfer $(n=112)$ and FET $(n=330)$. In this study, we used the spontaneously conceived group as the reference group.

The mean maternal age was $32.58 \pm 5.12$ years, and 1250 $(52.6 \%)$ women were nulliparous, and the mean prepregnancy BMI was $21.42 \pm 3.73 \mathrm{~kg} / \mathrm{m}^{2}$. Table 1 lists the clinical characteristics of the study population.

There were differences in maternal age, birth weight, intrapartum haemorrhage, nulliparity, caesarean section, HDP, GDM, hypothyroidism, family history of hypertension and family history of diabetes mellitus in the four groups.

Solutions for fixed effects of intrauterine growth analyses and intrauterine growth trajectories of male fetuses

Solutions for the fixed effects of intrauterine growth analyses are presented in table 2. For the effect of infertility treatment as a primary outcome, only the interaction between infertility treatment (FET) and gestation duration (estimate: $0.36 ; 95 \%$ CI: 0.06 to 0.67 ) significantly affected the intrauterine growth of male fetuses. The association between additional factors and male intrauterine growth was also investigated. The following factors significantly affected the intrauterine growth of male fetuses; multiparity (estimate: -35.71 ; $95 \%$ CI: -65.63 to -5.80 ), interaction between multiparity and gestation duration (estimate: 0.23 ; 95\% CI: 0.02 to 0.44 ), HDP (estimate: 82.22; $95 \%$ CI: 8.49 to 155.96$)$, interaction between HDP and gestation duration (estimate: -0.56 ; 95\% CI: -1.06 to 0.064 ), prepregnancy BMI (underweight) (estimate: 78.91; 95\% CI: 38.09 to 119.73), prepregnancy BMI (overweight) (estimate: -79.45 ; 95\% CI: -122.21 to -36.69 ), 
Table 1 Maternal background characteristics in FET, fresh-embryo transfer, infertility treatment without ART and spontaneously conceived groups

\begin{tabular}{|c|c|c|c|c|c|}
\hline $\begin{array}{l}\text { Characteristics of } \\
\text { the women }\end{array}$ & FET group $(n=330)$ & $\begin{array}{l}\text { Fresh-embryo } \\
\text { transfer group } \\
(n=112)\end{array}$ & $\begin{array}{l}\text { Infertility treatment } \\
\text { without ART group } \\
(n=171)\end{array}$ & $\begin{array}{l}\text { Spontaneously } \\
\text { conceived group } \\
\text { ( } n=1764)\end{array}$ & $P$ value \\
\hline Maternal age (years) & $35.92 \pm 3.84$ & $36.00 \pm 3.95$ & $34.33 \pm 4.28$ & $31.57 \pm 5.07$ & $<0.001$ \\
\hline $\begin{array}{l}\text { Gestational period } \\
\text { (days) }\end{array}$ & $270.68 \pm 20.17$ & $269.39 \pm 16.77$ & $270.99 \pm 15.70$ & $269.65 \pm 20.08$ & 0.71 \\
\hline Birth weight (g) & $3063.34 \pm 500.77$ & $2882.30 \pm 393.12$ & $3035.82 \pm 472.20$ & $2975.84 \pm 494.86$ & $<0.001$ \\
\hline $\begin{array}{l}\text { Intrapartum } \\
\text { haemorrhage (g) }\end{array}$ & $898.17 \pm 738.95$ & $602.47 \pm 428.90$ & $669.61 \pm 612.61$ & $584.14 \pm 441.80$ & $<0.001$ \\
\hline $\begin{array}{l}\text { Prepregnancy BMI } \\
\left(\mathrm{kg} / \mathrm{m}^{2}\right)\end{array}$ & $21.34 \pm 2.77$ & $21.07 \pm 2.93$ & $21.63 \pm 3.30$ & $21.44 \pm 3.97$ & 0.64 \\
\hline Nulliparity & $223(67.5)$ & $86(76.8)$ & $113(66.1)$ & 828 (46.9) & $<0.001$ \\
\hline Caesarean section & $122(36.9)$ & $29(25.9)$ & $50(29.2)$ & $498(28.2)$ & 0.03 \\
\hline HDP & $21(6.4)$ & $6(5.4)$ & $10(5.8)$ & $56(3.2)$ & 0.03 \\
\hline GDM & $4(1.2)$ & $0(0.0)$ & $1(0.6)$ & $48(2.7)$ & 0.04 \\
\hline Previa placenta & $2(0.6)$ & $1(0.9)$ & $2(1.2)$ & $18(1.0)$ & 0.90 \\
\hline Basedow disease & $6(1.8)$ & $2(1.8)$ & $3(1.8)$ & $17(0.9)$ & 0.44 \\
\hline Hypothyroidism & $14(4.2)$ & $4(3.6)$ & $7(4.1)$ & $35(2.0)$ & 0.04 \\
\hline $\begin{array}{l}\text { Smoking during } \\
\text { pregnancy }\end{array}$ & $2(0.6)$ & $0(0.0)$ & $2(1.2)$ & $40(2.3)$ & 0.07 \\
\hline Family history of HT & $91(27.6)$ & $30(26.8)$ & $52(30.4)$ & $355(20.1)$ & $<0.001$ \\
\hline Family history of DM & $78(23.6)$ & $21(18.8)$ & $42(24.6)$ & $278(15.8)$ & $<0.001$ \\
\hline
\end{tabular}

Values are presented as average \pm SD or number (\%).

ART, assisted reproductive technology; BMI, body mass index; DM, diabetes mellitus; FET, frozen embryo transfer; GDM, gestational diabetes mellitus; HDP, hypertensive disorders of pregnancy; HT, hypertension.

interaction between prepregnancy BMI (underweight) and gestation duration (estimate: -0.53 ; 95\% CI: -0.81 to -0.24 ), interaction between prepregnancy BMI (overweight) and gestation duration (estimate: $0.48 ; 95 \% \mathrm{CI}$ : 0.18 to 0.77 ), and GDM (estimate: $-111.30 ; 95 \% \mathrm{CI}$ : -195.63 to -26.95$)$.

The intrauterine growth trajectories of male fetuses are shown in figure 1. It can be presumed that there was little difference in the intrauterine growth during the first trimester in all groups, and only the FET group showed more intrauterine growth compared with the reference group from the second trimester to term. EFW calculated at 37 weeks was $2650 \mathrm{~g}$ in the reference group and $2709 \mathrm{~g}$ in the FET group.

Solutions for fixed effects of intrauterine growth analyses and intrauterine growth trajectories of female fetuses

Solutions for the fixed effects of intrauterine growth analyses are presented in table 3 . We found that infertility treatment (FET) (estimate: $-69.85 ; 95 \%$ CI: -112.09 to -27.61$)$ and the interaction between infertility treatment (FET) and gestation duration (estimate: 0.57; 95\% CI: 0.28 to 0.87 ) significantly affected intrauterine growth of the female fetus. The following factors significantly affected the intrauterine growth of female fetuses; prepregnancy BMI (underweight) (estimate: 85.50;
95\% CI: 47.96 to 123.05$)$, interaction between prepregnancy BMI (underweight) and gestational duration (estimate: -0.57 ; $95 \%$ CI: -0.82 to -0.31 ), Basedow disease (estimate: 120.76 ; 95\% CI: 5.08 to 236.43), interaction between Basedow disease and gestation duration (estimate: $-0.85 ; 95 \%$ CI: -1.65 to -0.05 ), and GDM (estimate: -170.01 ; $95 \%$ CI: -270.01 to -70.00$)$.

The intrauterine growth trajectories are shown in figure 2. Similar to male fetuses, it can be presumed that there is a slight difference in the intrauterine growth in the first trimester in all groups and only the FET group showed more intrauterine growth than the reference group from the second trimester to term. This difference was more significant in female than in male fetuses. The EFW calculated at 37 weeks was $2614 \mathrm{~g}$ in the reference group and $2693 \mathrm{~g}$ in the FET group.

\section{DISCUSSION}

This study evaluated the association between infertility treatment and intrauterine growth using multilevel analyses and described intrauterine growth trajectories using EFW. In both sexes, only the FET group gained more intrauterine growth than the spontaneously conceived 
Table 2 Solutions for fixed effects for estimated fetal weight based on the multivariate regression model after controlling for gestational duration, maternal age, parity, infertility treatment, prepregnancy BMI, smoking status, pregnancy complications and their interaction in male neonates

\begin{tabular}{|c|c|c|c|}
\hline Factor & Male & & \\
\hline & Estimate & SE & $P$ value \\
\hline Intercept & -674.94 & 432.7 & 0.12 \\
\hline Gestational duration & 7.48 & 4.29 & 0.08 \\
\hline Period & & & $<0.0001$ \\
\hline Period 1 & 0 & Reference & Reference \\
\hline Period 2 & 78.56 & 435.27 & 0.87 \\
\hline Period 3 & -553.07 & 435.59 & 0.2 \\
\hline Period 4 & -1540.12 & 435.16 & 0.0004 \\
\hline Period 5 & -2417.04 & 435.42 & $<0.0001$ \\
\hline Period 6 & -2818.34 & 435.84 & $<0.0001$ \\
\hline Period 7 & -2704.81 & 436.13 & $<0.0001$ \\
\hline Period 8 & -2425.97 & 473.7 & $<0.0001$ \\
\hline Period*gestational duration & & & $<0.0001$ \\
\hline Period $1^{*}$ gestational duration & 0 & Reference & Reference \\
\hline Period $2^{*}$ gestational duration & -1.15 & 4.31 & 0.79 \\
\hline Period $3^{*}$ gestational duration & 3.61 & 4.30 & 0.40 \\
\hline Period $4^{*}$ gestational duration & 9.71 & 4.30 & 0.02 \\
\hline Period $5^{\star}$ gestational duration & 14.37 & 4.30 & 0.0008 \\
\hline Period 6*gestational duration & 16.23 & 4.30 & 0.0002 \\
\hline Period $7^{*}$ gestational duration & 15.8 & 4.29 & 0.0002 \\
\hline Period $8^{*}$ gestational duration & 14.73 & 4.34 & 0.0007 \\
\hline Multiparity & -35.71 & 15.26 & 0.02 \\
\hline Multiparity*gestational duration & 0.23 & 0.11 & 0.03 \\
\hline HDP & 82.22 & 37.62 & 0.03 \\
\hline $\mathrm{HDP}^{*}$ gestational duration & -0.56 & 0.26 & 0.03 \\
\hline Infertility treatment & & & 0.03 \\
\hline Spontaneously conceived & 0 & Reference & Reference \\
\hline Infertility treatment without ART & 123.63 & 65.32 & 0.06 \\
\hline Fresh-embryo transfer & 55.89 & 42.08 & 0.18 \\
\hline FET & -34.54 & 22.2 & 0.12 \\
\hline Infertility treatment ${ }^{\star}$ gestational duration & & & 0.01 \\
\hline Spontaneously conceived*gestational duration & 0 & Reference & Reference \\
\hline Infertility treatment without $A R T^{\star}$ gestational duration & -0.84 & 0.46 & 0.07 \\
\hline Fresh-embryo transfer*gestational duration & -0.28 & 0.29 & 0.33 \\
\hline FET*gestational duration & 0.36 & 0.16 & 0.02 \\
\hline Maternal age & & & 0.9 \\
\hline $25-34$ & 0 & Reference & Reference \\
\hline$<25$ & 14.11 & 32.55 & 0.66 \\
\hline$\geq 35$ & -1.34 & 16.05 & 0.93 \\
\hline Maternal age ${ }^{\star}$ gestational duration & & & 0.44 \\
\hline $25-34^{*}$ gestational duration & 0 & Reference & Reference \\
\hline$<25$ years* ${ }^{*}$ estational duration & -0.28 & 0.22 & 0.22 \\
\hline$\geq 35$ years* ${ }^{\star}$ estational duration & 0.019 & 0.11 & 0.87 \\
\hline
\end{tabular}

Continued 


\begin{tabular}{|c|c|c|c|}
\hline \multirow[t]{2}{*}{ Factor } & \multicolumn{3}{|l|}{ Male } \\
\hline & Estimate & SE & $P$ value \\
\hline Prepregnancy BMI & & & $<0.0001$ \\
\hline Normal & 0 & Reference & Reference \\
\hline Underweight & 78.91 & 20.82 & 0.0002 \\
\hline Overweight & -79.45 & 21.81 & 0.0003 \\
\hline Prepregnancy BMI*gestational duration & & & $<0.0001$ \\
\hline Normalgestational duration & 0 & Reference & Reference \\
\hline Underweight*gestational duration & -0.53 & 0.14 & 0.0003 \\
\hline Overweight*gestational duration & 0.48 & 0.15 & 0.0017 \\
\hline Smoking & 79.61 & 53.17 & 0.13 \\
\hline Smoking*gestational duration & -0.45 & 0.37 & 0.22 \\
\hline Previa placenta & 26.86 & 83.71 & 0.75 \\
\hline Previa placenta*gestational duration & -0.12 & 0.52 & 0.82 \\
\hline Basedow disease & -88.79 & 65.62 & 0.18 \\
\hline Basedow disease*gestational duration & 0.74 & 0.45 & 0.1 \\
\hline Hypothyroidism & -24.37 & 45.67 & 0.59 \\
\hline Hypothyroidismgestational duration & 0.23 & 0.32 & 0.47 \\
\hline GDM & -111.3 & 43.02 & 0.0097 \\
\hline GDM $^{*}$ gestational duration & 0.87 & 0.3 & 0.0042 \\
\hline
\end{tabular}

ART, assisted reproductive technology; BMI, body mass index; FET, frozen embryo transfer; GDM, gestational diabetes mellitus; HDP, hypertensive disorders of pregnancy.

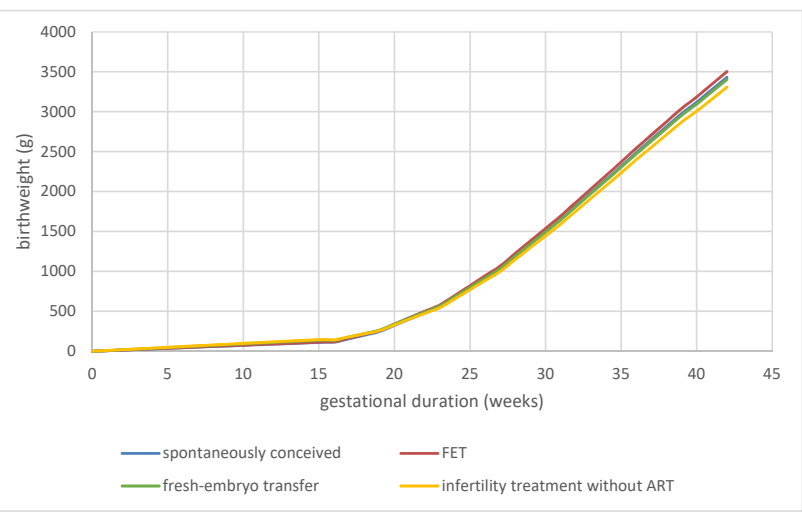

\begin{tabular}{|c|c|c|c|c|c|c|c|}
\hline Gestational duration (week) & 16 & 20 & 24 & 28 & 32 & 37 & 40 \\
\hline Spontaneously conceived (g) & 113 & 324 & 673 & 1192 & 1819 & 2650 & 3119 \\
\hline FET (g) & 119 & 340 & 699 & 1228 & 1865 & 2709 & 3186 \\
\hline Fresh-embryo transfer (g) & 136 & 340 & 681 & 1192 & 1811 & 2632 & 3096 \\
\hline $\begin{array}{c}\text { Infertility treatment } \\
\text { without ART (g) }\end{array}$ & 142 & 330 & 655 & 1150 & 1754 & 2556 & 3007 \\
\hline
\end{tabular}

Figure 1 Intrauterine growth estimated trajectories and estimated fetal weight at each gestational week depending on the type of infertility treatment of male fetuses. These trajectories are described using the estimated value of the reference group except for the type of infertility treatment (25-34 years old, nulliparity, normal prepregnancy BMI, non-smoking, no pregnancy complications). ART, assisted reproductive technology; BMI, body mass index; FET, frozen embryo transfer group from the second trimester to term. This difference was particularly significant in female fetuses.

To the best of our knowledge, this is the first study to evaluate the association between infertility treatment and intrauterine growth using multilevel analyses of longitudinal EFW data obtained at prenatal check-ups in Japanese patients.

Our result was consistent with that of a previous study, which showed that FET was associated with birth weights $192 \mathrm{~g}$ higher than those for spontaneously conceived neonates. ${ }^{8}$ In our multilevel model, the EFW at 40 weeks was estimated to be $90-124 \mathrm{~g}, 67-90 \mathrm{~g}$ and $110-179 \mathrm{~g}$ higher in the FET singletons than in freshembryo transfer singletons, spontaneously conceived singletons, and fertility treatment without ART singletons, respectively. This is due to the accumulation of growth differences between the FET group and other infertility treatment groups from the second trimester to term. There are several hypothetical mechanisms for the higher intrauterine growth rate seen after FET. First, the techniques of freezing and thawing enable the positive selection of high-quality embryos that survive the cryopreservation procedure. Therefore, it can be speculated that these embryos can also be more successful in other selective events, such as implantation, invasion and placentation or nutrition supplementation. ${ }^{56}{ }^{30}$ Second, FET performed in a natural cycle provides different conditions for embryo implantation and growth due to a 
Table 3 Solutions for fixed effects for estimated fetal weight based on the multivariate regression model after controlling for gestational duration, maternal age, parity, infertility treatment, prepregnancy BMI, smoking status, pregnancy complications and their interaction in female neonates

\begin{tabular}{|c|c|c|c|}
\hline \multirow[t]{2}{*}{ Factor } & \multicolumn{3}{|l|}{ Female } \\
\hline & Estimate & SE & $P$ value \\
\hline Intercept & -160.67 & 460.53 & 0.73 \\
\hline Gestational duration & 2.21 & 4.55 & 0.63 \\
\hline Period & & & $<0.0001$ \\
\hline Period 1 & 0 & Reference & Reference \\
\hline Period 2 & -419.29 & 463.13 & 0.37 \\
\hline Period 3 & -1004.82 & 463.49 & 0.03 \\
\hline Period 4 & -2020.79 & 463.03 & $<0.0001$ \\
\hline Period 5 & -2922.03 & 463.28 & $<0.0001$ \\
\hline Period 6 & -3485.55 & 463.82 & $<0.0001$ \\
\hline Period 7 & -3199.34 & 463.51 & $<0.0001$ \\
\hline Period 8 & -2883.19 & 496.27 & $<0.0001$ \\
\hline Period*gestational duration & & & $<0.0001$ \\
\hline Period $1{ }^{*}$ gestational duration & 0 & Reference & Reference \\
\hline Period $2^{*}$ gestational duration & 3.91 & 4.57 & 0.39 \\
\hline Period $3^{*}$ gestational duration & 8.32 & 4.56 & 0.07 \\
\hline Period $4^{*}$ gestational duration & 14.63 & 4.55 & 0.0013 \\
\hline Period $5^{\star}$ gestational duration & 19.41 & 4.55 & $<0.0001$ \\
\hline Period $6^{*}$ gestational duration & 22.03 & 4.55 & $<0.0001$ \\
\hline Period $7^{\star}$ gestational duration & 20.86 & 4.55 & $<0.0001$ \\
\hline Period $8^{\star}$ gestational duration & 19.65 & 4.59 & $<0.0001$ \\
\hline Multiparity & -16.27 & 14.47 & 0.26 \\
\hline Multiparity ${ }^{\star}$ gestational duration & 0.07 & 0.099 & 0.48 \\
\hline HDP & 43.1 & 41.43 & 0.3 \\
\hline HDP* $^{*}$ gestational duration & -0.23 & 0.28 & 0.43 \\
\hline Infertility treatment & & & 0.0049 \\
\hline Spontaneously conceived & 0 & Reference & Reference \\
\hline Infertility treatment without ART & 65.48 & 63.56 & 0.3 \\
\hline Fresh-embryo transfer & 17.67 & 37.45 & 0.64 \\
\hline FET & -69.85 & 21.54 & 0.0012 \\
\hline Infertility treatment* gestational duration & & & 0.0007 \\
\hline Spontaneously conceivedªestational duration & 0 & Reference & Reference \\
\hline Infertility treatment without $\mathrm{ART}^{*}$ gestational duration & -0.30 & 0.44 & 0.49 \\
\hline Fresh-embryo transfer*gestational duration & -0.18 & 0.26 & 0.48 \\
\hline FET*gestational duration & 0.57 & 0.15 & 0.0001 \\
\hline Maternal age & & & 0.34 \\
\hline 25-34 & 0 & Reference & Reference \\
\hline$<25$ & -44.82 & 30.47 & 0.14 \\
\hline$\geq 35$ & -5.77 & 15.35 & 0.71 \\
\hline Maternal age*gestational duration & & & 0.46 \\
\hline $25-34^{*}$ gestational duration & 0 & Reference & Reference \\
\hline$<25$ years* ${ }^{*}$ estational duration & 0.21 & 0.21 & 0.32 \\
\hline$\geq 35$ years $^{*}$ gestational duration & 0.1 & 0.11 & 0.37 \\
\hline
\end{tabular}

Continued 


\begin{tabular}{|c|c|c|c|}
\hline \multirow{2}{*}{$\begin{array}{l}\text { Factor } \\
\text { Prepregnancy BMI }\end{array}$} & \multicolumn{3}{|l|}{ Female } \\
\hline & & & $<0.0001$ \\
\hline Normal & 0 & Reference & Reference \\
\hline Underweight & 85.5 & 19.15 & $<0.0001$ \\
\hline Overweight & -39.71 & 21.69 & 0.07 \\
\hline Prepregnancy BMI*gestational duration & & & $<0.0001$ \\
\hline Normal*gestational duration & 0 & Reference & Reference \\
\hline Underweight* gestational duration & -0.57 & 0.13 & $<0.0001$ \\
\hline Overweight*gestational duration & 0.25 & 0.15 & 0.1 \\
\hline Smoking & -16.03 & 47.73 & 0.74 \\
\hline Smoking*gestational duration & 0.12 & 0.33 & 0.71 \\
\hline Previa placenta & 187.04 & 176.06 & 0.29 \\
\hline Previa placenta*gestational duration & -1.17 & 0.93 & 0.21 \\
\hline Basedow disease & 120.76 & 59.01 & 0.04 \\
\hline Basedow disease* gestational duration & -0.85 & 0.41 & 0.04 \\
\hline Hypothyroidism & -7.99 & 39.23 & 0.84 \\
\hline Hypothyroidismgestational duration & 0.095 & 0.27 & 0.73 \\
\hline GDM & -170.01 & 51.01 & 0.0009 \\
\hline GDM$^{*}$ gestational duration & 1.09 & 0.35 & 0.0022 \\
\hline
\end{tabular}

ART, assisted reproductive technology; BMI, body mass index; FET, frozen embryo transfer; GDM, gestational diabetes mellitus; HDP, hypertensive disorders of pregnancy.

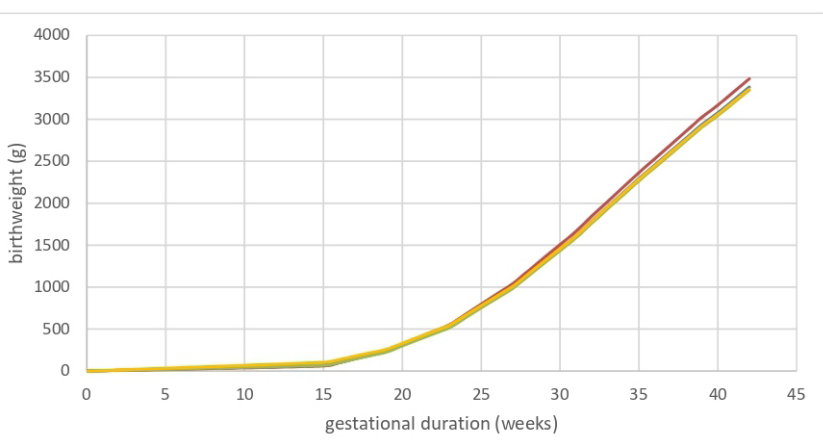

$\begin{array}{ll}\text { - spontaneously conceived } & \text { FET } \\ \text { fresh-embryo transfer } & \text { infertility treatment without ART }\end{array}$

\begin{tabular}{|c|c|c|c|c|c|c|c|}
\hline Gestational duration (week) & 16 & 20 & 24 & 28 & 32 & 37 & 40 \\
\hline Spontaneously conceived (g) & 105 & 308 & 649 & 1153 & 1784 & 2614 & 3077 \\
\hline FET (g) & 99 & 318 & 675 & 1196 & 1842 & 2693 & 3167 \\
\hline Fresh-embryo transfer (g) & 101 & 300 & 636 & 1135 & 1760 & 2584 & 3043 \\
\hline $\begin{array}{c}\text { Infertility treatment } \\
\text { without ART (g) }\end{array}$ & 136 & 331 & 663 & 1159 & 1782 & 2601 & 3057 \\
\hline
\end{tabular}

Figure 2 Intrauterine growth estimated trajectories and estimated fetal weight at each gestational week depending on the type of infertility treatment of female fetuses. These trajectories are described using the estimated value of the reference group except for the type of infertility treatment (25-34 years old, nulliparity, normal prepregnancy BMI, non-smoking, no pregnancy complications). ART, assisted reproductive technology; BMI, body mass index; FET, frozen embryo transfer. different hormonal environment compared with the stimulated cycle. ${ }^{5630}$ We could not accurately obtain detailed information on hormonal treatment due to the retrospective design of our study. Therefore, we could not assess this effect. Finally, the cryopreservation technique can induce changes in the embryo at the epigenetic level. ${ }^{30}$ Cryoprotectants affect DNA methylation and can provide protection against adverse effects on imprinting, ${ }^{6}$ and the practice of culturing frozen embryos overnight before transfer on day 2 or 3 means a more advanced cleavage stage in comparison with fresh embryos. ${ }^{6}$

We also found possible sex differences in the association between intrauterine growth and FET with a significant association for the female sex. Only a few studies as of current date have focused on this association between intrauterine growth and sex differences with IVF pregnancies. O'Neill et al reported that conception via IVF does not enhance sex-dependent growth differences. ${ }^{31}$ This study by $\mathrm{O}^{\prime} \mathrm{Neill}$ et al had certain limitations as compared with our study, such as relatively small sample size and no information on the difference between FET and fresh-embryo transfer. Conversely, Keane et al reported that the effect of FET birth weight was only significant for female infants. ${ }^{32}$ The mechanisms leading to sexdependent differences in intrauterine growth is not clear. One possible explanation could be that male and female neonates employ different mechanisms to cope with adverse environments or events, such as maternal asthma and pre-eclampsia. ${ }^{31} 33$ The FET process introduces a 
number of potential stressors including freezing and thawing. Sex differences in response to these stresses may lead to a difference in intrauterine growth. Therefore, in this study, we analysed male and female fetuses separately. Further studies are required to better understand this relationship.

Our study has several strengths. We performed a multilevel analysis with a relatively large sample size considering the characteristics of longitudinal EFW at multiple prenatal check-ups throughout gestation. Most previous studies that focused on intrauterine growth used data from at most two or three prenatal check-ups and related birth weights. ${ }^{35}$ In our study, we assessed intrauterine growth differences according to infertility treatment by adding a time-dependent covariate to the multilevel model.

This study has certain limitations. First, since it's a single-centre study, it might be difficult to extrapolate our results to the general population. The CI of the study results was relatively wide because of the small number of participants who received fertility treatment. Although we performed this multilevel analysis in a relatively large sample of pregnant women, it may be necessary to recruit more participants, especially by fertility treatment, to obtain appropriate results. A study based on population-based databases such as the ART database of the Japan Society of Obstetrics and Gynaecology would help confirm these results. ${ }^{4}$ Second, data on other factors which may affect intrauterine growth, such as gestational weight gain, intake of alcohol and caffeine, antiphospholipid syndrome, differences in culture medium, preeclampsia, egg donation and socioeconomic status ${ }^{13}$ 37-40 could not be assessed accurately because of the retrospective nature of the study. Additionally, egg donation is not a common practice in Japan. Some participants in this study may have the aforementioned risk factors. There might be selection bias on the socio-economic status between each category in particular since ART in Japan is expensive and not covered by public health insurance. Third, smoking status and GDM information could be inaccurate. In comparison to previous studies, ${ }^{41}{ }^{42}$ this study might have underestimated maternal smoking and GDM because of several possible reasons. For instance, we used a questionnaire to ascertain maternal smoking status instead of using objective measurements. The smoking status was recorded as a dichotomous response, that is, 'smoking' included those participants who only answered 'smoking during pregnancy' and 'non-smoking' included those who answered 'have quit smoking', 'have never smoked', or 'no answer'. For unclear reasons, there were some missing data about GDM on the electronic record. However, data on other variables are likely to be relatively more accurate and objective compared with the previous studies. ${ }^{43-45}$ GDM was significantly associated with intrauterine growth regardless of sex despite an underestimation of the incidence of GDM. While it is necessary to confirm the incidence of GDM in future studies, we believe that its underestimation in this study did not adversely affect the results. Fourth, the EFW used for the evaluation of intrauterine growth was not actual fetal weight; therefore, actual fetal growth might have been assessed inaccurately. However, previous studies that have evaluated the accuracy of sonographic fetal weight have reported high validity of ultrasonic estimation of fetal weight. ${ }^{46-49}$ Barel et al reported that the Shinozuka technique used for evaluation of EFW in this study has a high coefficient of correlation (0.91) between EFW and actual birth weight. ${ }^{48}$ Therefore, we believe that EFW is an accurate measure of intrauterine growth that can reliably assess differences among the different types of infertility treatments. Finally, the longer the gestational period, the more likely it is that the fetus would be born via vaginal delivery or caesarean section. In other words, the number of EFW to be analysed decreases as it approaches the expected date of delivery. Thus, the possibility of a type II error might increase, especially in the third trimester. Although missing data can be handled flexibly in multilevel analysis, the results might have been influenced by missing values of EFW in the third trimester.

In summary, this study showed that intrauterine growth from the second trimester to term differs significantly between women who conceived via FET compared with those who conceived spontaneously. Considering the difference in intrauterine growth from the early stage in the FET group, the results support the mechanism for the higher intrauterine growth observed after FET (ie, positive selection for freezing). This difference was most notable in female fetuses. We believe that our research question is novel and will trigger further prospective research, both basic and clinical, to examine the effect of infertility treatment on fetal growth.

Acknowledgements We thank the study subjects for the use of their personal data.

Contributors SS: data collection. SS, SH, KS: conception or design of the work; data analysis and interpretation; critical revision of the article; drafting of the article and final approval of the version to be published.

Funding A part of this study was supported by Grants-in-Aid for Scientific Research (KAKENHI 15K08731 and 18K10076) from the Ministry of Education, Culture, Sports, Science and Technology (MEXT), Japan, and Grant-in-Aid for Scientific Research (17gk0110013h00002) on the Japan Agency for Medical Research and Development (AMED).

Competing interests None declared.

Patient and public involvement Patients and/or the public were not involved in the design, or conduct, or reporting, or dissemination plans of this research.

Patient consent for publication Not required.

Ethics approval The study design was reviewed and approved by the Human Subjects Review Committee of the University of Yamanashi (number 1681: 2017). All procedures were performed in accordance with the 1964 Declaration of Helsinki and its later amendments.

Provenance and peer review Not commissioned; externally peer reviewed.

Data availability statement All data relevant to the study are included in the article or uploaded as supplementary information.

Open access This is an open access article distributed in accordance with the Creative Commons Attribution Non Commercial (CC BY-NC 4.0) license, which permits others to distribute, remix, adapt, build upon this work noncommercially, and license their derivative works on different terms, provided the original work is properly cited, appropriate credit is given, any changes made 
indicated, and the use is non-commercial. See: http://creativecommons.org/ licenses/by-nc/4.0/.

ORCID iDs

Satoshi Shinohara http://orcid.org/0000-0003-3124-1141

Kohta Suzuki http://orcid.org/0000-0002-8151-6927

\section{REFERENCES}

1 Kamel RM. Management of the infertile couple: an evidence-based protocol. Reprod Biol Endocrinol 2010;8:21.

2 de Mouzon J, Goossens V, Bhattacharya S, et al. Assisted reproductive technology in Europe, 2006: results generated from European registers by ESHRE. Hum Reprod 2010;25:1851-62.

3 Pereira N, Rosenwaks Z, A fresh RZ. A fresh(er) perspective on frozen embryo transfers. Fertil Steril 2016;106:257-8.

4 Saito H, Jwa SC, Kuwahara A, et al. Assisted reproductive technology in Japan: a summary report for 2015 by the ethics Committee of the Japan Society of obstetrics and gynecology. Reprod Med Biol 2018;17:20-8.

5 Pelkonen S, Koivunen R, Gissler M, et al. Perinatal outcome of children born after frozen and fresh embryo transfer: the Finnish cohort study 1995-2006. Hum Reprod 2010;25:914-23.

6 Pinborg A, Loft A, Aaris Henningsen A-K, et al. Infant outcome of 957 singletons born after frozen embryo replacement: the Danish nationa cohort study 1995-2006. Fertil Steril 2010;94:1320-7.

7 D'Angelo DV, Whitehead N, Helms K, et al. Birth outcomes of intended pregnancies among women who used assisted reproductive technology, ovulation stimulation, or no treatment. Fertil Steril 2011;96:314-20.

8 Valenzuela-Alcaraz B, Crispi F, Manau D, et al. Differential effect of mode of conception and infertility treatment on fetal growth and prematurity. J Matern Fetal Neonatal Med 2016;29:3879-84.

9 Maheshwari A, Raja EA, Bhattacharya S. Obstetric and perinatal outcomes after either fresh or thawed frozen embryo transfer: an analysis of 112,432 singleton pregnancies recorded in the human fertilisation and embryology authority anonymized dataset. Fertil Steril 2016;106:1703-8.

10 Roque M, Lattes K, Serra S, et al. Fresh embryo transfer versus frozen embryo transfer in in vitro fertilization cycles: a systematic review and meta-analysis. Fertil Steril 2013;99:156-62.

11 Shapiro BS, Daneshmand ST, Bedient CE, et al. Comparison of birth weights in patients randomly assigned to fresh or frozen-thawed embryo transfer. Fertil Steril 2016;106:317-21.

12 Swanson JM, Entringer S, Buss C, et al. Developmental origins of health and disease: environmental exposures. SeminReprod Med 2000;27:391-402.

13 Minakami H, Maeda T, Fujii T, et al. Guidelines for obstetrical practice in Japan: Japan Society of obstetrics and gynecology (JSOG) and Japan association of obstetricians and Gynecologists (JAOG) 2014 edition. J Obstet Gynaecol Res 2014;40:1469-99.

14 Chiavaroli V, Castorani V, Guidone P, et al. Incidence of infants born small- and large-for-gestational-age in an Italian cohort over a 20year period and associated risk factors. Ital J Pediatr 2016;42:42.

15 León G, Murcia M, Rebagliato M, et al. Maternal thyroid dysfunction during gestation, preterm delivery, and birthweight. The Infancia Y Medio Ambiente cohort, Spain. Paediatr Perinat Epidemiol 2015;29:113-22.

16 Hinkle SN, Albert PS, Mendola P, et al. Differences in risk factors for incident and recurrent small-for-gestational-age birthweight: a hospital-based cohort study. BJOG 2014;121:1080-9.

17 Yeniel AO, Ergenoglu AM, Itil IM, et al. Effect of placenta previa on fetal growth restriction and stillbirth. Arch Gynecol Obstet 2012;286:295-8.

18 Watanabe K, Matsubara K, Nakamoto O, et al. New definition and classification of "Hypertensive Disorders of Pregnancy (HDP)". Hypertens Res Pregnancy 2017;5:39-40.

19 Suzuki K, Sato M, Zheng W, et al. Childhood growth trajectories according to combinations of pregestational weight status and maternal smoking during pregnancy: a multilevel analysis. PLoS One 2015;10:e0118538.

20 Wendland EM, Torloni MR, Falavigna M, et al. Gestational diabetes and pregnancy outcomes--a systematic review of the World Health Organization (WHO) and the International Association of Diabetes in Pregnancy Study Groups (IADPSG) diagnostic criteria. BMC Pregnancy Childbirth 2012;12:23.

21 Oppenheimer L, Armson A, Farine D, MATERNAL FETAL MEDICINE COMMITTEE. Diagnosis and management of placenta previa. $J$ Obstet Gynaecol Can 2007;29:261-6.
22 Teng W, Shan Z, Patil-Sisodia K, et al. Hypothyroidism in pregnancy. Lancet Diabetes Endocrinol 2013;1:228-37.

23 Shinozuka N, Akamatsu N, Sato S, et al. Ellipse tracing fetal growth assessment using abdominal circumference: JSUM standardization Committee for fetal measurements. J Med Ultrasound 2000;8:87-94.

24 Vinkesteijn AS, Mulder PG, Wladimiroff JW. Fetal transverse cerebellar diameter measurements in normal and reduced fetal growth. Ultrasound Obstet Gynecol 2000;15:47-51.

25 Cohen E, Baerts W, van Bel F. Brain-sparing in intrauterine growth restriction: considerations for the neonatologist. Neonatology 2015;108:269-76.

26 Kwok O-M, Underhill AT, Berry JW, et al. Analyzing longitudinal data with multilevel models: an example with individuals living with lower extremity intra-articular fractures. Rehabil Psychol 2008;53:370-86

27 Zheng W, Suzuki K, Sato M, et al. Adolescent growth in overweight and non-overweight children in Japan: a multilevel analysis. Paediatr Perinat Epidemiol 2014;28:263-9.

28 Zheng W, Suzuki K, Yokomichi H, et al. Multilevel longitudinal analysis of sex differences in height gain and growth rate changes in Japanese school-aged children. J Epidemiol 2013;23:275-9.

29 Suzuki K, Kondo N, Sato M, et al. Gender differences in the association between maternal smoking during pregnancy and childhood growth trajectories: multilevel analysis. Int $J$ Obes 2011;35:53-9.

30 Korosec S, Frangez HB, Steblovnik L, et al. Independent factors influencing large-for-gestation birth weight in singletons born after in vitro fertilization. J Assist Reprod Genet 2016;33:9-17.

31 O'Neill KE, Tuuli M, Odibo AO, et al. Sex-Related growth differences are present but not enhanced in in vitro fertilization pregnancies. Fertil Steril 2014;101:407-12.

32 Keane KN, Mustafa KB, Hinchliffe P, et al. Higher $\beta$-hCG concentrations and higher birthweights ensue from single vitrified embryo transfers. Reprod Biomed Online 2016;33:149-60.

33 Murphy VE, Gibson PG, Giles WB, et al. Maternal asthma is associated with reduced female fetal growth. Am J Respir Crit Care Med 2003;168:1317-23.

34 Stark MJ, Dierkx L, Clifton VL, et al. Alterations in the maternal peripheral microvascular response in pregnancies complicated by preeclampsia and the impact of fetal sex. J Soc Gynecol Investig 2006;13:573-8.

35 Broere-Brown ZA, Baan E, Schalekamp-Timmermans S, et al. SexSpecific differences in fetal and infant growth patterns: a prospective population-based cohort study. Biol Sex Differ 2016;7:65.

36 Gaillard R, Steegers EA, de Jongste JC, et al. Tracking of fetal growth characteristics during different trimesters and the risks of adverse birth outcomes. Int J Epidemiol 2014;43:1140-53.

37 Harita N, Kariya M, Hayashi T, et al. Gestational bodyweight gain among underweight Japanese women related to small-forgestational-age birth. J Obstet Gynaecol Res 2012;38:1137-44.

38 Räisänen S, Gissler M, Sankilampi U, et al. Contribution of socioeconomic status to the risk of small for gestational age infants-a population-based study of 1,390,165 singleton live births in Finland. Int J Equity Health 2013;12:28.

39 Figueras F, Gratacós E. Update on the diagnosis and classification of fetal growth restriction and proposal of a stage-based management protocol. Fetal Diagn Ther 2014;36:86-98.

40 Jeve YB, Potdar N, Opoku A, et al. Donor oocyte conception and pregnancy complications: a systematic review and meta-analysis. BJOG 2016;123:1471-80.

41 Kampmann U, Madsen LR, Skajaa GO, et al. Gestational diabetes: a clinical update. World J Diabetes 2015;6:1065-72.

42 Suzuki K, Shinohara R, Sato M, et al. Association between maternal smoking during pregnancy and birth weight: an appropriately adjusted model from the Japan environment and children's study. J Epidemiol 2016;26:371-7.

43 Umesawa M, Kobashi G. Epidemiology of hypertensive disorders in pregnancy: prevalence, risk factors, predictors and prognosis. Hypertens Res 2017;40:213-20.

44 Lazarus $\mathrm{JH}$. Epidemiology and prevention of thyroid disease in pregnancy. Thyroid 2002;12:861-5.

45 Matsuda Y, Hayashi K, Shiozaki A, et al. Comparison of risk factors for placental abruption and placenta previa: case-cohort study. $J$ Obstet Gynaecol Res 2011;37:538-46.

46 Kurmanavicius J, Burkhardt T, Wisser J, et al. Ultrasonographic fetal weight estimation: accuracy of formulas and accuracy of examiners by birth weight from 500 to 5000 G. J Perinat Med 2004;32:155-61.

47 Melamed N, Yogev Y, Meizner I, et al. Sonographic fetal weight estimation: which model should be used? J Ultrasound Med 2009;28:617-29. 
48 Barel O, Vaknin Z, Tovbin J, et al. Assessment of the accuracy of multiple sonographic fetal weight estimation formulas: a 10 -year experience from a single center. $J$ Ultrasound Med 2013;32:815-23.
49 Faschingbauer F, Raabe E, Heimrich J, et al. Accuracy of sonographic fetal weight estimation: influence of the scan-to-delivery interval in combination with the applied weight estimation formula. Arch Gynecol Obstet 2016;294:487-93. 